\title{
Desenvolvimento de Instrumentação para Geração de Nuvem de Pontos Usando Sensores Inerciais e LiDAR
}

\author{
Judá T. Santos* Marcus D. N. Forte* Adriano R. Paula* \\ Nadson T. Souza * Thiago A. Lima* Magno Prudêncio* \\ Italo C. Branco ${ }^{* *}$ Fabricio G. Nogueira* Bismark C. Torrico* \\ Wilkley B. Correira* \\ * Departamento de Engenharia Elétrica, Universidade Federal do \\ Ceará, Av. Mister Hull - Pici - 60455-760 (e-mails: \\ juda-santos@alu.ufc.br; davi2812@dee.ufc.br; rodrigues@dee.ufc.br; \\ nadson.tome@dee.ufc.br; thiago.lima@alu.ufc.br; \\ magnoprudencio@alu.ufc.br; fnogueira@dee.ufc.br; bismark@dee.ufc.br; \\ wilkley@dee.ufc.br) \\ ** EDP Brasil - PPTM \\ São Gonçalo do Amarante, Ceará, Brasil (e-mail: \\ italo.alves@edpenergiapecem.com.br)
}

\begin{abstract}
This works presents the development of an embedded system which generates a point cloud data based on measurements acquired by LiDAR (Light Detection and Ranging) sensors. The LiDAR sensor is integrated with an IMU (Inertial Measuring Unit) so that angular motion is detected and synchronized with the measured distances, forming a three-dimensional point cloud. A mathematical model based on quaternions is used for the angular motion estimation.

Resumo: Este artigo descreve o desenvolvimento de um sistema embarcado para visão computacional em nuvem de pontos utilizando fusão sensorial entre sensor LiDAR (Light Detection and Ranging) e dados inerciais. O desenvolvimento apresenta a descrição dos equipamentos utilizados e o projeto de um Filtro de Kalman Estendido para a aplicação da técnica. A seção de resultados contempla imagens obtidas por experimentos em campo e análise do desempenho do sistema.
\end{abstract}

Keywords: Embedded Systems; Point cloud; Sensor Fusion.

Palavras-chaves: Sistemas embarcados; Nuvem de Pontos; Fusão Sensorial

\section{INTRODUÇÃO}

Diante do crescente uso de sensores para mapeamento de regiões e geolocalização, e a complexidade dos casos de estudo, faz-se necessário o estudo e desenvolvimento de equipamentos que sejam eficazes em dois importantes fatores: aquisição de dados do ambiente e precisão no posicionamento. A eficiência de um sistema de aquisição de dados junto do geoposicionamento pode ser visto em trabalhos como no mapeamento de grandes áreas abertas Casagli et al. (2017), na modelagem de áreas urbanas Vacca et al. (2018), no desenvolvimento de sistemas de transportes autônomos Zhang et al. (2018), na mineração $\mathrm{Hu}$ and $\mathrm{Wu}$ (2016), dentre outros.

\footnotetext{
* À Coordenação de Aperfeiçoamento de Pessoal de Nível Superior (CAPES) e a Fundação Cearense de Apoio ao Desenvolvimento Científico e Tecnológico (Funcap), pelo apoio durante o projeto. À EDP Energia Pecém e ao programa de Pesquisa e Desenvolvimento Tecnológico do Setor de Energia Elétrica da ANEEL por todo apoio recebido durante a elaboração e execução do projeto PD-07267$0016 / 2018$
}

Uma das maneiras de se obter a posição e orientação de um robô móvel é utilizando técnicas SLAM (Simultaneous Localization and Mapping), Li et al. (2016), para mapeamento de posição em ambientes fechados, e as Unidades de Medidas Inerciais, conhecido no inglês como IMU (Inertial Measurement Unit). Estes sensores inerciais podem ser constituídos de acelerômetro, giroscópio e magnetômetro. Assim, com essas informações era possível rastrear o caminho percorrido pelo veículo, Pivarčiova et al. (2018).

Outra ferramenta utilizada é o sensor LiDAR (Light Detection and Ranging). Esta tecnologia é utilizada para captação de dados de uma região através de nuvem de pontos, Fortin et al. (2015). Esses dados são tratados de acordo com o interesse de estudo, podendo ser aplicados também em diversas áreas. Como exemplo, o sistema LiDAR pode ser implementado no mapeamento de zonas florestais Wallace et al. (2012), no controle de posição em carro autônomo Meng et al. (2017), na inspeção de tubulação Guerreiro et al. (2018); Kumar et al. (2017), dentre outros.

Alguns problemas podem ser observados nesses equipamentos. Sensores inerciais por si só podem apresentar 
imprecisões e interferências nos dados obtidos. Estas imprecisões podem acarretar em uma leitura errada do sensor LiDAR, gerando assim, um conjunto de pontos que não condizem com área ou objeto mapeado. Com finalidade de se evitar isto, técnicas de fusão sensorial utilizando Filtro de Kalman são desenvolvidas, onde essas interferências são levadas em consideração na modelagem do sistema e amenizadas Forte (2018).

Assim, a problemática tratada neste artigo é de construir um sistema de aquisição de dados preciso e compacto para fazer o mapeamento de uma região. As informações obtidas pelos sensores é tratada através da fusão sensorial entre o sistema IMU e o LiDAR utilizando técnicas como Filtro de Kalman para atenuar possíveis imprecisões. Todos os dados obtidos são tratados através de uma Raspberry $3 B+$ contendo ROS (Robot Operating System), sistema base para todos os algoritmos de comunicação e processamento de nuvem de pontos do sistema apresentado.

O artigo é dividido nas seguintes seções e seus temas: Seção 2 apresenta a instrumentação e os sensores utilizados neste projeto; Seção 3 contém a fusão de dados inerciais e do sensor LiDAR; Seção 4 apresenta os resultados de testes experimentais; Seção 5 conclui o artigo com uma conclusão dos resultados obtidos.

\section{INSTRUMENTAÇÃO E SENSORES}

\subsection{LiDAR}

O sensor LiDAR é um sistema de aquisição de distâncias por emissão e recepção de feixes de luz. Este é considerado um dos sensores mais apropriados para aplicações que demandam precisão na aquisição de dados espaciais para captação de formas e superfícies Kotb et al. (2018).

Seu princípio de funcionamento se assemelha ao de um sensor de distância a laser convencional. Entretanto, o LiDAR emite um feixe de luz que faz uma coleta de vários pontos vizinhos em seu campo de visão, fazendo uma varredura em um eixo cartesiano. Os dados são amostrados na forma de vetores que retornam valores de distâncias. Para formar uma nuvem de pontos, é necessário realizar novas leituras subsequentes que capturam seções vizinhas entre si e concatenar os vetores de distância.

As duas principais formas de aquisição dessa nuvem de pontos consistem na modificação do processo de varredura ou por um passo angular ou por um deslocamento do sensor sob um eixo cartesiano diferente do eixo de varredura.

\subsection{IMU}

Sensores inerciais são comumente referidos como um conjunto composto de um acelerômetro e um giroscópio cujas medidas são dadas nos três eixos locais ao sensor. Acelerômetros são capazes de medir acelerações próprias de um corpo, ou seja, acelerações relativas a um corpo em queda livre. O fato de acelerômetros capturarem o efeito da aceleração da gravidade é fundamental para o projeto de sistemas estimação de orientação de corpos móveis, uma vez que o vetor gravidade é constante enquanto o quadro móvel gira. Por essa razão este vetor passa a ser referência para um sistema de estimação da orientação.
Os giroscópios são capazes de medir velocidades angulares referenciadas a um quadro móvel. Também é comum que algumas IMUs possuam sensores magnéticos, ou magnetômetros. Estes sensores medem o vetor intensidade do campo magnético sobre o qual o sensor está sujeito referentes ao quadro móvel do sensor.

Contudo, nenhum sensor está livre de ruídos, vieses, erros de escalonamento ou não linearidades. Pequenos vieses no giroscópio fariam com que a integração das velocidades gerasse erros de deriva. Acelerômetros e magnetômetros são conhecidos por possuírem considerável ruído de medição. São esses efeitos indesejados que a teoria de Fusão Sensorial visa mitigar. Dentre as diversas técnicas para fusão de dados da literatura Lee et al. (2009), destaca-se aqui o uso do filtro de Kalman para fusão de dados.

A grande vantagem do uso de filtros de Kalman é sua abordagem estocástica que permite a inclusão das características dos ruídos dos sensores na modelagem do filtro. Além disso, por ser um filtro ótimo para sistemas lineares Friedland (1985), seu uso é bastante aconselhado. Existem também versões não-lineares do filtro de Kalman, conhecidos como filtro de Kalman estendido Julier and Uhlmann (1997), ou EKF (Extended Kalman Filter), e o filtro de Kalman Unscented Wan and Van Der Merwe (2000), ou UKF (Unscented Kalman Filter), que introduzem soluções sub-ótimas ao problema da filtragem não-linear.

\subsection{Sistema embarcado}

A plataforma portátil desenvolvida neste projeto conta com a integração do sensor LiDAR, a unidade de medição inercial e um sistema embarcado para analise dos dados obtidos. O modelo de sensor LiDAR utilizado foi o LD-MRS420201S01, que consegue captar informações em longas distâncias, dependendo do tipo de material a ser observado, e com ângulo de abertura de até $110^{\circ}$, o que auxilia na captação de informações em grandes áreas ou objetos.

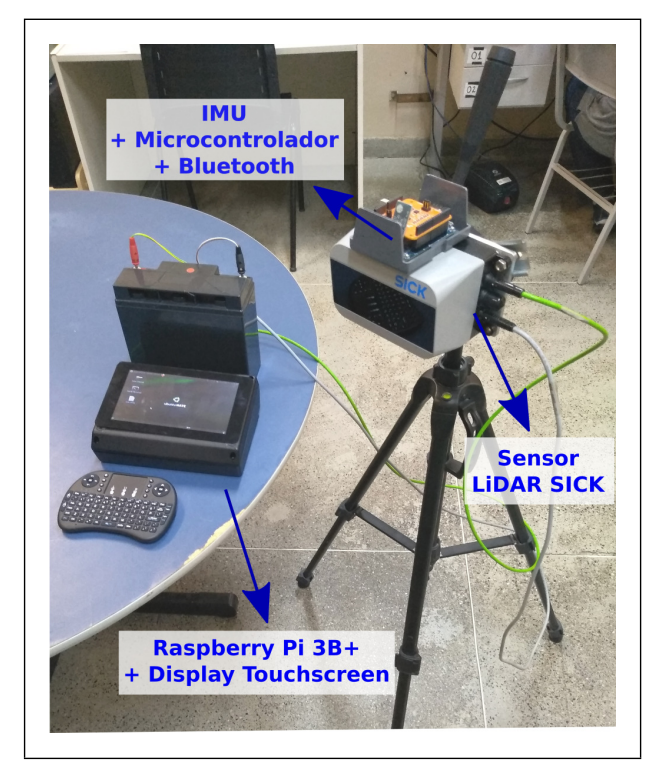

Figura 1. Plataforma móvel.

O sistema IMU aplicado neste projeto tem a capacidade de fornecer a orientação tridimensional do sensor, ou seja, 
é capaz de fornecer efetivamente os ângulos de rolagem (roll), arfagem (pitch) e guinada (yaw). Isso é possível devido a implementação de filtros não-lineares e quatérnions, elevando a precisão e a rapidez com que a IMU fornece os ângulos. O módulo IMU é composto por um microcontrolador FRDM-K64F com processador ARM ${ }^{\circledR}$ Cortex ${ }^{\circledR}$ M4, junto do sensor inercial FRDM-STBC-AGM01 e um módulo de comunicação Bluetooth.

O módulo IMU é acoplado diretamente ao sensor LiDAR, como pode ser visto na Figura 1. O conjunto é então instalado em um tripé que permite a movimentação do conjunto no eixo horizontal e no vertical, o que permite a varredura $3 \mathrm{D}$ de grandes áreas.

Com a finalidade analisar e processar todas as informações obtidas tanto pelo sensor LiDAR quanto pelo sistema inercial a utilização de um computador se faz necessária. O computador embarcado utilizado é o Raspberry Pi 3B+. Este modelo é capaz de ser host do framework de robótica, ROS. Sendo este utilizado na fusão sensorial e na formação da nuvem de pontos adquiridas pelos sensores através de algoritmos dedicas a isto.

Este modelo também apresenta capacidade de transmissão via conexão Ethernet, por onde recebe as informações do LiDAR e tecnologia Bluetooth 4.2, utilizada para comunicação com o módulo IMU. Além de possuir conexão para interface touchscreen. Devido seu tamanho compacto e sua capacidade de processamento, este computador embarcado torna-se fundamental para a proposta do artigo.

Por fim, o sistema é alimentado por uma bateria de $12 \mathrm{~V}$ que alimenta o sensor LiDAR quanto o módulo IMU. O esquemático reduzido do sistema desenvolvido é apresentado na Figura 2.

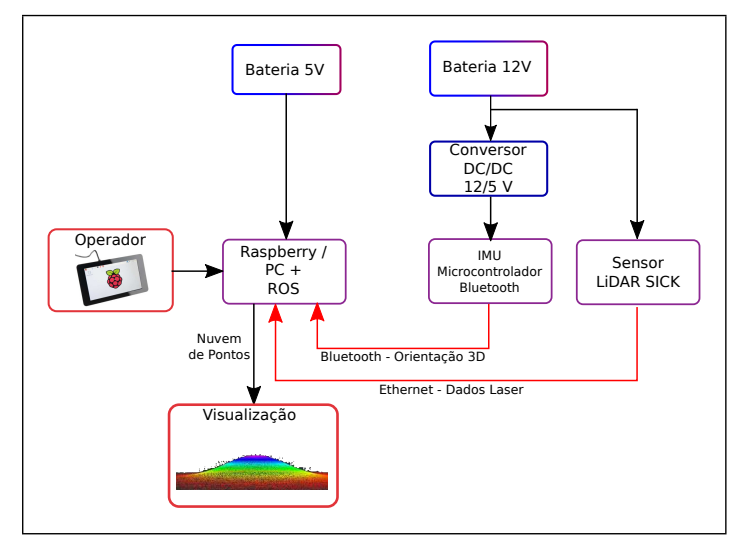

Figura 2. Diagrama do sistema.

\section{FUSÃO DE DADOS INERCIAIS E LIDAR}

A fusão de dados é feita combinando a estimação de orientação dos dados da IMU sincronizadas com o vetor de varredura laser do LiDAR. A combinação dos dados é feita através do ROS, que possui bibliotecas para conversão e transformações dos dados LiDAR.

Duas transformações são aplicadas sobre a leitura dos dados, uma de rotação e outra de translação. A transformação de rotação é descrita por um quatérnion, e a transformação de translação é descrita pelas coordenadas cardinais do sensor lidar. Neste estudo, é considerado o caso de o sensor não sofrer deslocamentos translacionais, apenas rotacionais. Dessa forma, a estimação precisa da orientação do sensor é fundamental.

\subsection{Estimação da orientação}

A orientação de sistemas móveis pode ser descrita através da matemática de quatérnions. Seu uso é favorecido em comparação com a abordagem clássica dos ângulos de Euler, pois esta última possui singularidades nas matrizes de rotação quando os ângulos se aproximam de $\frac{\pi}{2}$. Neste trabalho é utilizada a abordagem quaterniônica. Um quatérnion é um número que possui três partes imaginárias e uma parte real:

$$
q=q_{0}+q_{1} i+q_{2} j+q_{3} k=\left(q_{0}, \boldsymbol{q}_{\boldsymbol{i}}\right),
$$

no qual $\boldsymbol{q}_{\boldsymbol{i}}=\left[\begin{array}{lll}q_{1} & q_{2} & q_{3}\end{array}\right]^{T}$ é a parte imaginária na forma de vetor $\mathbb{R}^{3}$. Além disso, é dado que:

$$
i^{2}=j^{2}=k^{2}=-1 \quad i j=k, j k=i, k i=j .
$$

O quatérnion pode ser usado para expressão da rotação de corpos rígidos. Através de operações matemáticas definidas no seu domínio complexo, é possível descrever rotações de quadros ou de vetores. A descrição de um vetor $v$ no quadro móvel $b$ para o quadro de navegação/inercial $n$, por exemplo, é calculada por:

$$
v_{n}=q_{b}^{n} \otimes v_{b} \otimes\left(q_{b}^{n}\right)^{-1},
$$

cujo produto quaterniônico é definido algebricamente por:

$$
q \otimes p=\left[\begin{array}{cccc}
q_{0} & -q_{1} & -q_{2} & -q_{3} \\
q_{1} & q_{0} & -q_{3} & q_{2} \\
q_{2} & q_{3} & q_{0} & -q_{1} \\
q_{3} & -q_{2} & q_{1} & q_{0}
\end{array}\right]\left[\begin{array}{c}
p_{0} \\
p_{1} \\
p_{2} \\
p_{3}
\end{array}\right]
$$

A Eq. (3) pode ser utilizada também para rotacionar um vetor $v_{b}$ em torno de um eixo definido por $q_{b}^{n}$. Semelhantemente, descreve-se a transformação inversa da Eq. (3), dada por:

$$
v_{b}=\left(q_{b}^{n}\right)^{-1} \otimes v_{n} \otimes q_{b}^{n} .
$$

A Eq. (5) tem utilidade na conversão de vetores referentes a um quadro de navegação/inercial $n$ para o quadro móvel b. Um exemplo seria a conversão dos vetores da gravidade e campo magnético da terra, cujos valores são conhecidos no quadro de navegação $n$.

Descreve-se agora o modelo da estimação do quatérnion de rotação do sistema. Define-se a velocidade medida pelo giroscópio referentes ao seu quadro móvel dada por:

$$
\omega_{b}=\left[\begin{array}{lll}
\omega_{x} & \omega_{y} & \omega_{z}
\end{array}\right]^{T}+\left[\beta_{x} \beta_{y} \beta_{z}\right]^{T} .
$$

onde, $\omega_{i}$ a velocidade angular real do eixo móvel $i$ e $\beta_{i}$ é o viés na medição da velocidade daquele eixo. 
A taxa de variação do quatérnion está relacionada com a velocidade angular através de Nonami et al. (2010)

$$
\dot{q}_{b}^{n}(t)=\frac{1}{2} q_{b}^{n}(t) \otimes \omega_{b q}(t),
$$

ou, no tempo discreto com tempo de amostragem $T_{s}$ :

$$
q_{b}^{n}(k+1)=q_{b}^{n}(k)+\frac{T_{s}}{2} q_{b}^{n}(k) \otimes \omega_{b q}(k),
$$

onde, $q_{b}^{n}$ é o quatérnion que representa a transformação de quadros, $\omega_{b q}$ é a representação quaterniônica do velocidades angulares referentes quadro móvel $b$, ou seja:

$$
\omega_{b q}=\left(0, \omega_{b}\right)
$$

Sabe-se que um giroscópio é capaz de fornecer vetor $\boldsymbol{\omega}_{\boldsymbol{b}}$, porém, corrompido com vieses de medição e ruídos. Como é desejado estimar e remover tais efeitos indesejados do sistema, define-se então o modelo de estimação $f$ cujo estado é o próprio quatérnion de rotação $x(k)=q_{b}^{n}(k)$ e sua entrada são as velocidades angulares giroscópicas $u(k)=\omega_{b q}(k)$.

$$
x(k+1)=f(x(k), u(k))+\eta_{f}
$$

Substituindo a 8 em 10, tem-se que:

$$
q_{b}^{n}(k+1)=q_{b}^{n}(k)+\frac{T_{s}}{2} q_{b}^{n}(k) \otimes \omega_{b q}(k)+\eta_{f} .
$$

Eq. (10) compõe o modelo não-linear de estimação do quatérnion de rotação de quadro $b$ para $n$. Nesse estudo, os vieses e ruídos do giroscópio são modelados em $\eta_{k}$ como uma distribuição gaussiana de média nula. Define-se agora o modelo de medições dos outros sensores disponíveis para medição. Sejam os vetores gravidade e campo magnético da terra referenciada ao eixo de navegação/inercial $g_{n}=$ $\left[\begin{array}{lll}0 & 0 & g\end{array}\right]^{T}$ e $b_{n}=\left[\begin{array}{lll}m & 0 & n\end{array}\right]^{T}$, onde $g$ é a aceleração da gravidade na superfície da terra, $m$ e $n$ são as componentes horizontal e vertical da inclinação magnética local. O acelerômetro e magnetômetro medem os vetores $g_{n}$ e $b_{n}$, porém, referenciados ao quadro móvel $b$, descritos por $z=\left[g_{b}+\Delta g b_{b}+\Delta b\right]^{T}$, juntamente com alguns efeitos não desejados $\Delta$ que podem conter acelerações externas, vieses ou erros de calibração. A função $h$ que descreve a leitura dos sensores é dada por:

$$
\begin{aligned}
z & =h(x(k))+\eta_{h}, \\
{\left[\begin{array}{l}
g_{b} \\
b_{b}
\end{array}\right] } & =\left[\begin{array}{l}
\left(q_{b}^{n}\right)^{-1} \otimes g_{n} \otimes q_{b}^{n} \\
\left(q_{b}^{n}\right)^{-1} \otimes b_{n} \otimes q_{b}^{n}
\end{array}\right]+\left[\begin{array}{c}
\Delta g \\
\Delta b
\end{array}\right] .
\end{aligned}
$$

Nesse caso, os efeitos indesejados são modelados como ruído branco gaussiano de média nula $\eta_{h}=\left[\begin{array}{c}\Delta g \\ \Delta b\end{array}\right]$.

\subsection{Projeto do Filtro de Kalman Estendido}

Um dos objetivos deste estudo é a estimação precisa do quatérnion $q_{b}^{n}$. Uma vez que seu modelo é um sistema não-linear, optou-se pela utilização do filtro de Kalman Estendido. O algoritmo do filtro pode ser dividido em duas etapas, de predição e de atualização, segundo os passos: Predição
(1) $\hat{x}_{k}^{-}=f\left(\hat{x}_{k-1}, u_{k-1}\right)$
(2) $P_{k}^{-}=F_{k} P_{k-1} F_{k}^{T}+Q_{k}$

\section{Atualização}

(3) $K_{k}=P_{k}^{-} H_{k}\left(H_{k} P_{k}^{-} H_{k}^{T}+R_{k}\right)^{-1}$

(4) $\hat{x}_{k}=\hat{x}_{k}^{-}+K_{k}\left(z_{k}-h\left(\hat{x}_{k}^{-}\right)\right)$

(5) $P_{k}=\left(I-K_{k} H_{k}\right) P_{k}^{-}$

onde $\hat{x}_{k}$ são os estados estimados, $z_{k}$ é a saída medida $P_{k}$ é a matriz de covariância do erro dos estados, $Q_{k}$ é a matriz de covariância do processo, $R_{k}$ é a matriz de covariância da saída. As funções $f$ e $h$ são dadas pelo sistema, que no caso em análise, são as Eqs (10) e (13), respectivamente. Portanto, basta agora calcular as jacobianas.

$$
\begin{aligned}
& F_{k}=\left.\frac{\partial f}{\partial x}\right|_{\hat{x}_{k-1}, u_{k}}, \quad H_{k}=\left.\frac{\partial h}{\partial x}\right|_{\hat{x}_{k}^{-}}=\left[\begin{array}{c}
H_{k}^{1} \\
H_{k}^{2}
\end{array}\right] \\
& F_{k}=\left[\begin{array}{cccc}
1 & -\frac{T_{s}}{2} \omega_{x}(k) & -\frac{T_{s}}{2} \omega_{y}(k) & -\frac{T_{s}}{2} \omega_{z}(k) \\
\frac{T_{s}}{2} \omega_{x}(k) & 1 & \frac{T_{s}}{2} \omega_{z}(k) & -\frac{T_{s}}{2} \omega_{y}(k) \\
\frac{T_{s}}{2} \omega_{y}(k) & -\frac{T_{s}}{2} \omega_{z}(k) & 1 & \frac{T_{s}}{2} \omega_{x}(k) \\
\frac{T_{s}}{2} \omega_{z}(k) & \frac{T_{s}}{2} \omega_{y}(k) & -\frac{T_{s}}{2} \omega_{x}(k) & 1
\end{array}\right] \\
& H_{k}^{1}=2\left[\begin{array}{cccc}
0 & 0 & 0 & 0 \\
-q_{b 2}^{n}(k) & q_{b 3}^{n}(k) & -q_{b 0}^{n}(k) & q_{b 1}^{n}(k) \\
q_{b 1}^{n}(k) & q_{b 0}^{n}(k) & q_{b 3}^{n}(k) & q_{b 2}^{n}(k) \\
q_{b 0}^{n}(k) & -q_{b 1}^{n}(k) & q_{b 2}^{n}(k) & q_{b 3}^{n}(k)
\end{array}\right] \\
& H_{k}^{2}=2 m\left[\begin{array}{cccc}
0 & 0 & 0 & 0 \\
q_{b 0}^{n}(k) & q_{b 1}^{n}(k) & -q_{b 2}^{n}(k) & -q_{b 3}^{n}(k) \\
-q_{b 3}^{n}(k) & q_{b 2}^{n}(k) & q_{b 1}^{n}(k) & -q_{b 0}^{n}(k) \\
q_{b 2}^{n}(k) & q_{b 3}^{n}(k) & q_{b 0}^{n}(k) & q_{b 1}^{n}(k)
\end{array}\right]+ \\
& 2 n\left[\begin{array}{cccc}
0 & 0 & 0 & 0 \\
-q_{b 2}^{n}(k) & q_{b 3}^{n}(k) & -q_{b 0}^{n}(k) & q_{b 1}^{n}(k) \\
q_{b 1}^{n}(k) & q_{b 0}^{n}(k) & q_{b 3}^{n}(k) & q_{b 2}^{n}(k) \\
q_{b 0}^{n}(k) & -q_{b 1}^{n}(k) & -q_{b 2}^{n}(k) & q_{b 3}^{n}(k)
\end{array}\right]
\end{aligned}
$$

De posse das matrizes jacobianas e das funções dos estados e medições o filtro de Kalman Estendido é implementado no sistema físico. As matrizes $Q_{k}$ e $R_{k}$ são obtidas experimentalmente pela leitura dos sensores enquanto inertes e obtendo a variância dos sinais. O filtro foi embarcado na placa de desenvolvimento FRDM-K64F com a ajuda das bibliotecas de processamento de sinais e operações matriciais.

\section{RESULTADOS EXPERIMENTAIS}

A plataforma de aquisição de dados desenvolvida possibilita a portabilidade do sistema para ambientes externos ou internos. Além disso, o sistema de referência de posicionamento, no caso a IMU, possibilita resultados bastante precisos da nuvem de pontos.

O primeiro ensaio consiste no teste da estabilidade do sistema durante uma aquisição de dados. Neste teste foi 


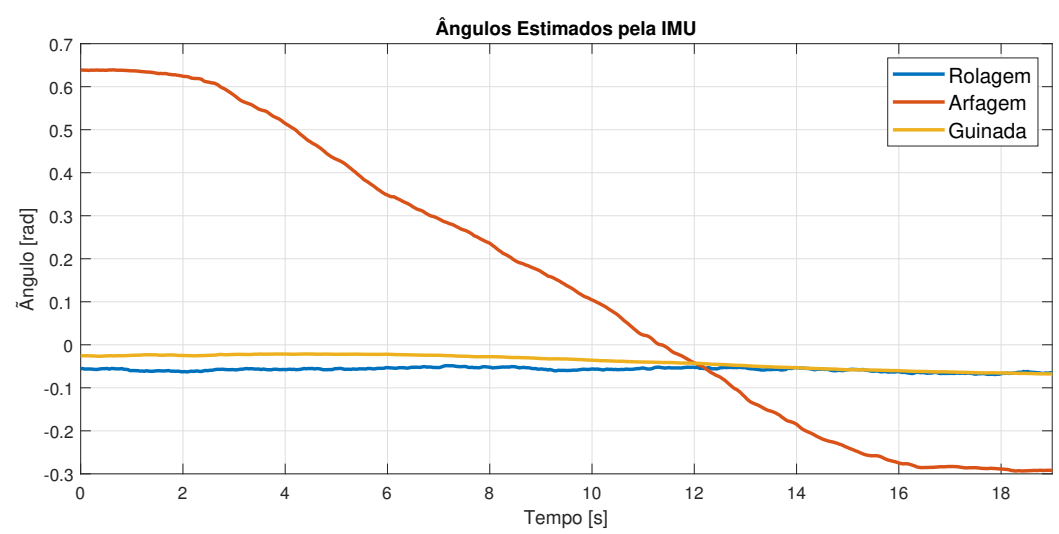

(a) Ângulos estimados

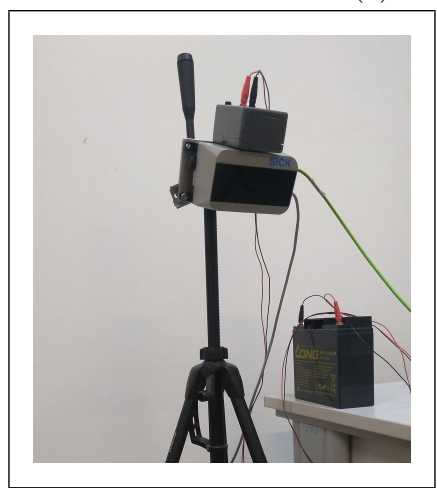

(b) Posição Inicial

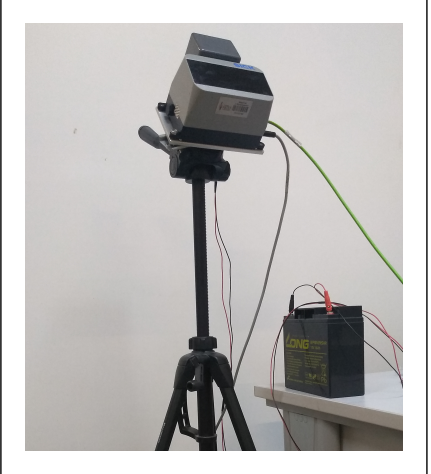

(c) Posição Final

Figura 3. Teste de estimação dos ângulos.

simulado uma leitura na vertical, iniciando com o sensor apontado para o solo, Figura $3 \mathrm{~b}$ e movido à posição final conforme a Figura 3c. A Figura 3a apresenta a variação dos três ângulos fornecidos pela IMU com relação ao tempo. Os ângulos são obtidos através da conversão do quatérnion estimado em ângulos de Euler. No ensaio é possível ver a variação apenas do ângulo de arfagem enquanto os outros ângulos permanecem com pequenas oscilações provenientes da trepidação do movimento.

O ensaio seguinte consiste na aquisição dados de uma área externa composta por diferentes tipos de materiais e vegetação. Nas Figuras 4a e 4b têm-se as comparações da foto do local de aquisição dos dados com a construção da nuvem de pontos gerada pelo sistema desenvolvido. Uma melhor perspectiva da nuvem de pontos gerada pode ser vista no vídeo de referência, https://youtu.be/9XHq1Nlj0Fw.

A imagem representada na Figura 4 b foi captada no sentido horizontal e faz possível notar que a profundidade da imagem de acordo com o seguinte código de cores: vermelho para objetos mais próximos, azul para os mais distante e o restante do espectro representa as distâncias intermediárias. A resolução do LiDAR permite que sejam reconhecidas as formas de árvores, paredes e postes demonstrando o seu potencial para identificação de formas e contornos.

Outro ensaio de campo é realizado para aferir maiores distâncias e testar a precisão de leitura do sistema de aquisição sob essa condição. A Figura 5 a traz a representação fotográfica do ambiente analisado. As Figuras 5b

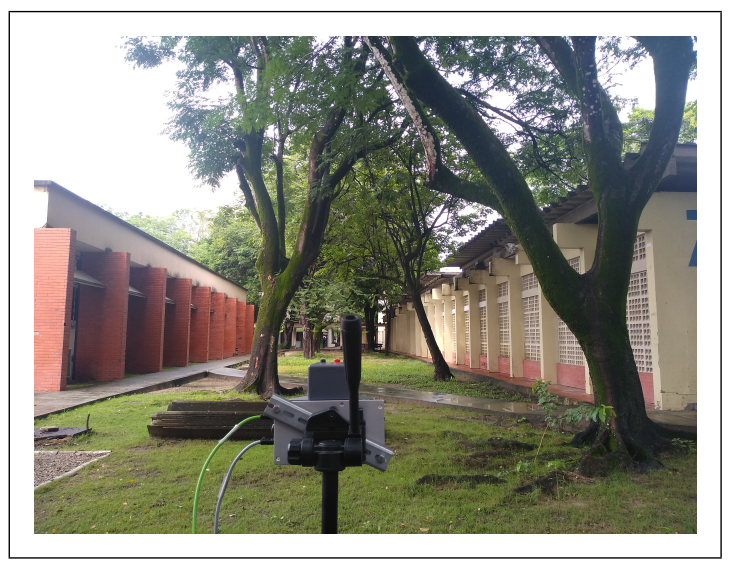

(a) Espaço de teste 1.

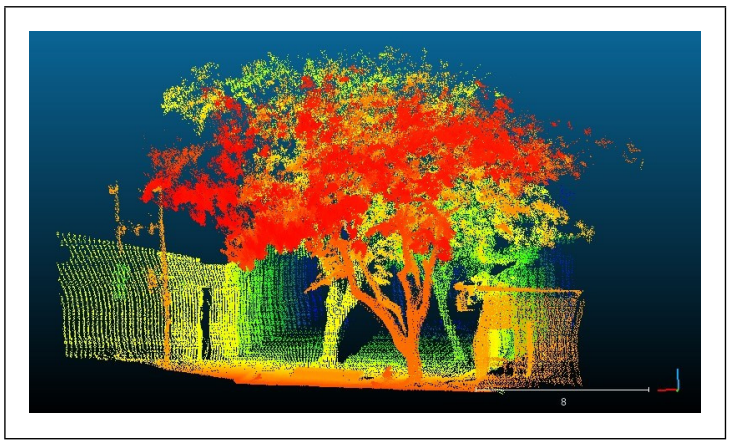

(b) Nuvem de pontos resultante.

Figura 4. Comparativo entre espaço e nuvem 3D. 
e 5 c mostram perspectivas diferentes da foto nas quais a manipulação da nuvem de pontos permite visualizar.

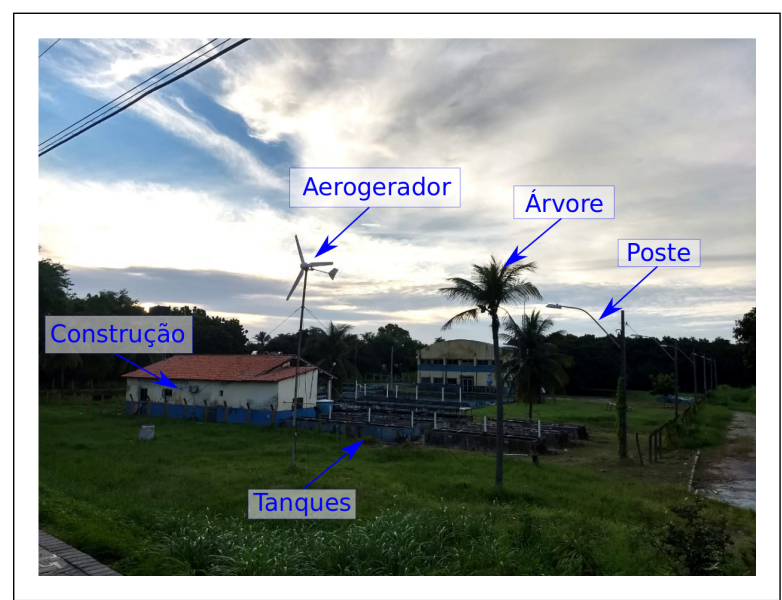

(a) Espaço de teste 2.

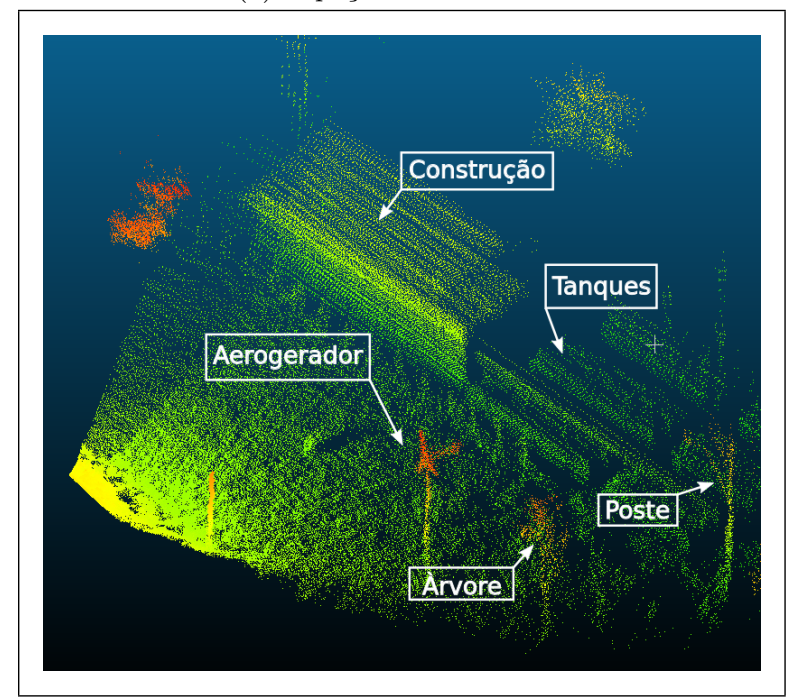

(b) Visão superior.

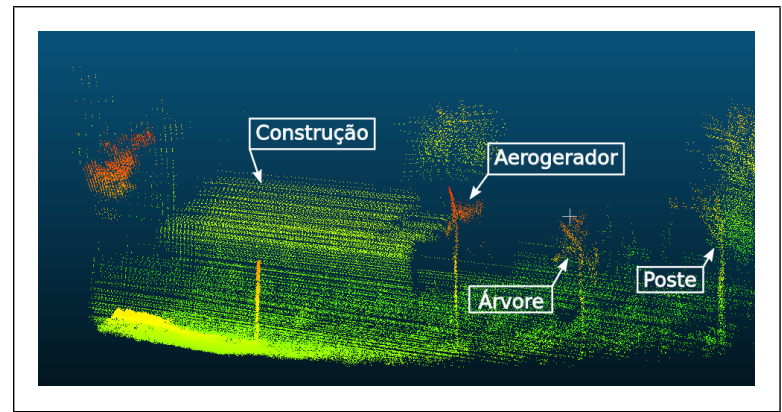

(c) Visão frontal.

Figura 5. Diferentes perspectivas da nuvem de pontos do espaço de teste 2 .

As leituras das Figuras 5b e $5 \mathrm{c}$ devem ser interpretadas de acordo com o seguinte código de cores: vermelho níveis mais altos, azul - níveis mais baixos, outras cores do espectro representam níveis intermediários de altura. O sensor LiDAR conseguiu captar com precisão as formas de objetos a mais de $90 \mathrm{~m}$ de distância. A superfície do campo e suas saliências podem ser melhor visualizadas na perspectiva apresentada na Figura 5b. É possível identificar com precisão na perspectiva apresentada na Figura $5 \mathrm{c}$ a forma de um gerador eólico também visto originalmente na Figura 5a. O manuseio destas perspectivas e destes diferentes códigos de cores é útil para a interpretação dos dados das nuvens de pontos.

\section{CONCLUSÕES}

Em conclusão a este trabalho, foi possível observar resultados satisfatórios na aquisição da variação dos ângulos obtidos pelo módulo IMU. Onde através da plataforma móvel foi possível reproduzir um movimento apenas na horizontal, sem interferir em outras variações angulares.

Também foi possível analisar a qualidade da aquisição da nuvem de pontos devido ao georreferenciamento da plataforma. Onde, a fusão sensorial aplicada permitiu que os vetores de distâncias de cada varredura do LiDAR fossem concatenados adequadamente retornando uma representação confiável dos ambientes analisados. Por fim, o sistema apresentado neste artigo se mostrou eficiente tanto na aquisição de dados quanto no processamento da nuvem de pontos $3 \mathrm{D}$ obtidas.

\section{AGRADECIMENTOS}

Ao Grupo de Pesquisa em Automação, Controle e Robótica (GPAR) do Departamento de Engenharia Elétrica da Universidade Federal do Ceará.

\section{REFERÊNCIAS}

Casagli, N., Frodella, W., Morelli, S., Tofani, V., Ciampalini, A., Intrieri, E., Raspini, F., Rossi, G., Tanteri, L., and Lu, P. (2017). Spaceborne, uav and ground-based remote sensing techniques for landslide mapping, monitoring and early warning. Geoenvironmental Disasters. doi:10.1186/s40677-017-0073-1.

Forte, M.D.D.N. (2018). Reference Trajectory Tracking Control of a Nonholonomic Mobile Robot with Inertial Sensor Fusion. Master's thesis, Universidade Federal do Ceará

Fortin, B., Lherbier, R., and Noyer, J.C. (2015). A modelbased joint detection and tracking approach for multivehicle tracking with lidar sensor. IEEE Transactions on Intelligent Transportation Systems, 16(4). doi:10.1109/ TITS.2015.2391131.

Friedland, B. (1985). Control Systems Design: An Introduction to State-Space Methods. McGraw-Hill Higher Education.

Guerreiro, B.J., Silvestre, C., Cunha, R., and Cabecinhas, D. (2018). Lidar-based control of autonomous rotorcraft for the inspection of pierlike structures. IEEE Transactions on Control Systems Technology, 26, 1430-1438. doi:10.1109/TCST.2017.2705058.

$\mathrm{Hu}, \mathrm{W}$. and Wu, L. (2016). Ground deformation extraction using visible images and lidar data in mining area. International Archives of the Photogrammetry, Remote Sensing and Spatial Information Sciences, 505-512. doi: 10.5194/isprsarchives-XLI-B7-505-2016.

Julier, S.J. and Uhlmann, J.K. (1997). A new extension of the kalman filter to nonlinear systems. 182-193.

Kotb, A., Hassan, S., and Hassan, H.E.Z. (2018). A comparative study among various algorithms for lossless 
airborne lidar data compression. 2018 14th International Computer Engineering Conference (ICENCO), 1721.

Kumar, G.A., Patil, A.K., Patil, R., Park, S.S., and Chai, Y.H. (2017). A lidar and imu integrated indoor navigation system for uavs and its application in realtime pipeline classification. Sensors. doi:10.3390/ s17061268.

Lee, S., Ko, H., and Hahn, H. (2009). Multisensor Fusion and Integration for Intelligent Systems: An Edition of the Selected Papers from the IEEE International Conference on Multisensor Fusion ... Springer Publishing Company, Incorporated, 1st edition.

Li, J., Zhong, R., Hu, Q., and Ai, M. (2016). Featurebased laser scan matching and its application for indoor mapping. Sensors. doi:10.3390/s16081265.

Meng, X., Wang, H., and Liu, B. (2017). A robust vehicle localization approach based on gnss/imu/dmi/lidar sensor fusion for autonomous vehicles. Sensors, 1-19. doi:10.3390/s17092140.

Nonami, K., Kendoul, F., Suzuki, S., Wang, W., and Nakazawa, D. (2010). Autonomous Flying Robots: Unmanned Aerial Vehicles and Micro Aerial Vehicles. Springer Publishing Company, Incorporated, 1st edition.

Pivarčiova, E., Božek, P., Turygin, Y., Zajačko, I., Shchenyatsky, A., Štefan Václav, Císar, M., and Gemela, B. (2018). Reviews on various inertial measurement unit (imu) sensor applications. International Journal of Advanced Robotic Systems. doi:10.1177/ 1729881418755165.

Vacca, G., Furfaro, G., and Dessi, A. (2018). The use of the uav images for the building $3 \mathrm{~d}$ model generation. The International Archives of the Photogrammetery, Remote Sensing and Spatial Information Sciences, 217-223.

Wallace, L., Lucieer, A., Watson, C., and Turner, D. (2012). Development of a uav-lidar system with application to forest inventory. Remote Sensing, 1519-1543.

Wan, E.A. and Van Der Merwe, R. (2000). The unscented kalman filter for nonlinear estimation. In Proceedings of the IEEE 2000 Adaptive Systems for Signal Processing, Communications, and Control Symposium (Cat. No.00EX373), 153-158. doi:10.1109/ASSPCC. 2000.882463.

Zhang, Y., Zhang, G., Fierro, R., and Yang, Y. (2018). Force-driven traffic simulation for a future connected autonomous vehicle-enabled smart transportation system. The Transactions on Intelligent Transportation Systems. 